\section{Interstitial lung abnormalities and self-reported health and functional status}

\section{ABSTRACT \\ We investigated the association between interstitial lung abnormalities (ILA) and self- reported measures of health and functional status in 5764 participants from the Age, Gene/Environment Susceptibility-Reykjavik study. The associations of ILA to activities of daily living (ADLs), general health status and physical activity were explored using logistic regression models. Participants with ILA were less likely to be independent in ADLs (OR 0.70; $95 \% \mathrm{Cl} 0.55$ to 0.90 ) to have good or better self-reported health (OR 0.66; 95\% Cl 0.52 to 0.82 ) and to participate in physical activity (OR $0.72 ; \mathrm{Cl} 0.56$ to 0.91$)$. The results demonstrate ILA's association with worsening self-reported health and functional status.}

\section{INTRODUCTION}

Interstitial lung abnormalities (ILA) includes a set of precisely defined CT changes, similar to those used to detect interstitial lung disease (ILD), but are often of a lesser magnitude. ${ }^{1-3}$ The argument for the relation of ILA to ILD has been strengthened by the association of ILA to several factors, including reductions in total lung capacity and an increase in mortality. ${ }^{134}$ Other notable associations of ILA include increased age, decreased diffusion capacity, in addition to shortness of breath with exertion, and chronic cough. ${ }^{125}$ Despite these associations, little is known about the relationship between ILA and self-reported measures of health and functional status.

\section{METHODS}

To explore these associations, data were obtained from the Age, Gene/Environment Susceptibility-Reykjavik (AGES-Reykjavik) study. Details of the study design have been previously published. ${ }^{6}$ Informed consent was obtained from all participants. Two separate CT scans of the thorax were obtained with a four-row CT scanner (Sensation, Siemens Medical System, Erlangen, Germany) that covered about $95 \%$ of the lungs, omitting the superior portion of the apices. The axial images were evaluated by up to three readers (pulmonologists and chest radiologists) blinded to participant-specific information, using a sequential reading method as previously described. ${ }^{1}$ Scans labelled as ILA, indeterminate and 20\% of normal scans were read by two readers and the third reader provided majority opinion on discordantly labelled scans. ILA was defined as 'non-dependent changes affecting more than 5\% of any lung zone, including reticular or groundglass abnormalities, diffuse centrilobular nodularity, non-emphysematous cysts, honeycombing or traction bronchiectasis' while 'focal or unilateral ground-glass attenuation, focal or unilateral reticulation or patchy ground-glass abnormalities ( $<5 \%$ of any lung zone)' were defined as indeterminate changes. The data on smoking status, health status, activities of daily living (ADLs) and physical activity were obtained from participant questionnaires. ${ }^{6}$ The question regarding health status was 'In general, how would you say your health is?', with the answers ranging from 1 ('excellent') to 5 ('poor'). For statistical analyses, participants were categorised into two groups based on whether they perceived their health as 'good' or better. Thus, one group was composed of participants that perceived their health as 'good', 'very good' or 'excellent', while participants that perceived their health as 'poor' or 'fair' made up the other group. ADLs were assessed by asking participants whether they had difficulties performing the following five activities: bathing, dressing, walking from room to room, transferring out of bed/chair or eating. For statistical analyses, participants were grouped into two groups: people independent in all ADLs and people

Table 1 Baseline characteristics of participants

\begin{tabular}{|c|c|c|c|c|}
\hline Participants & No ILA $(n=3216,61 \%)$ & Indeterminate ILA status $(n=1726,32 \%)$ & ILA $(n=378,7 \%)$ & $P$ value \\
\hline Mean age $(95 \% \mathrm{Cl})$ & 75.9 (75.7 to 76.1$)$ & 77.4 (77.1 to 77.6$)$ & 77.8 (77.2 to 78.3$)$ & $3.4 \times 10^{-9}$ \\
\hline Women, $\mathrm{n}(\%)$ & $1910(59)$ & $962(56)$ & $172(46)$ & $3.1 \times 10^{-7}$ \\
\hline Mean BMI (95\% Cl) & 27.2 (27.0 to 27.3$)$ & 26.8 (26.6 to 27.0$)$ & 27.1 (26.6 to 27.5$)$ & 0.60 \\
\hline History of smoking, $\mathrm{n}(\%)$ & $1750(54)$ & $1021(59)$ & $271(72)$ & $1.1 \times 10^{-10}$ \\
\hline Median pack-years (IQR) & $0(0-16)$ & $2.5(0-22.5)$ & $11.0(0-28.5)$ & $3.5 \times 10^{-16}$ \\
\hline Current smoker, n (\%) & $374(12)$ & $205(12)$ & $69(18)$ & 0.0003 \\
\hline \multicolumn{5}{|l|}{ Activities of daily living } \\
\hline Independence in all five ADLs, $\mathrm{n}(\%)$ & $2422(75)$ & $1191(69)$ & $251(66)$ & \\
\hline Independence in four ADLs, $\mathrm{n}(\%)$ & $434(13)$ & $253(15)$ & $65(17)$ & \\
\hline Independence in three or less ADLs, $\mathrm{n}(\%)$ & $336(10)$ & $259(15)$ & $54(14)$ & \\
\hline \multicolumn{5}{|l|}{ Health status } \\
\hline Health described as 'excellent', $\mathrm{n}(\%)$ & $742(23)$ & $368(21)$ & $70(19)$ & \\
\hline Health described as 'very good', $\mathrm{n}(\%)$ & $480(15)$ & $225(13)$ & $38(10)$ & \\
\hline Health described as 'good', n (\%) & $1010(31)$ & $512(30)$ & $109(29)$ & \\
\hline Health described as 'fair', n (\%) & $813(25)$ & $503(29)$ & $128(34)$ & \\
\hline Health described as 'poor', n (\%) & $166(5)$ & $114(7)$ & $31(8)$ & \\
\hline \multicolumn{5}{|l|}{ Physical activity in the past 12 months } \\
\hline No or rare physical activity, $\mathrm{n}(\%)$ & $1914(60)$ & $1115(65)$ & $258(68)$ & \\
\hline Weekly physical activity, n (\%) & $1263(39)$ & $580(34)$ & $108(29)$ & \\
\hline
\end{tabular}

$P$ values comparing participants without ILA and participants with ILA.

ADLs, activities of daily living; BMI, body mass index; ILA, interstitial lung abnormalities. 
Table 2 Associations of ILA with measures of health

\begin{tabular}{|c|c|c|c|c|}
\hline & Unadjusted OR $(95 \% \mathrm{Cl})$ & $P$ value & Adjusted OR (95\% Cl) & $P$ value \\
\hline \multicolumn{5}{|c|}{ Independence in activities of daily living } \\
\hline No ILA & 1.00 & \multirow{2}{*}{0.0008} & 1.00 & \multirow{2}{*}{0.0051} \\
\hline ILA & $0.67(0.53$ to 0.85$)$ & & $0.70(0.55$ to 0.90$)$ & \\
\hline \multicolumn{5}{|c|}{ Health status perceived as good or better* } \\
\hline No ILA & 1.00 & \multirow{2}{*}{$4.0 \times 10^{-6}$} & 1.00 & \multirow{2}{*}{0.0003} \\
\hline ILA & $0.60(0.48$ to 0.75$)$ & & $0.66(0.52$ to 0.82$)$ & \\
\hline \multicolumn{5}{|c|}{ Participation in physical activity } \\
\hline No ILA & 1.00 & \multirow{2}{*}{0.0002} & 1.00 & \multirow{2}{*}{0.0076} \\
\hline ILA & $0.63(0.50$ to 0.80$)$ & & $0.72(0.56$ to 0.91$)$ & \\
\hline
\end{tabular}

Associations were estimated with logistic regression models. The adjusted models are adjusted for sex, body mass index, age, pack-years and current smoking status as covariates.

*This includes self-reports of health status perceived as 'good', 'very good' and 'excellent', as opposed to 'poor' or 'fair' ILA, interstitial lung abnormalities.

dependent in one or more ADLs. Physical activity was estimated by asking participants how many hours per week, during the last 12 months, they participated in moderate-vigorous intensity physical activity. The possible answers were never, rarely, every week but less than 1 hour, 1-3 hours per week, 4-7 hours per week and more than 7 hours per week. For statistical analyses, people were categorised into two groups: people who never or rarely participated in physical activity and those who did so at least weekly. Comparisons of baseline characteristics were done using $\mathrm{X}^{2}$ tests, $\mathrm{t}$-tests and Wilcoxon rank-sum tests as appropriate. The analysis of the association of ILA with measures of health and functional status was done using logistic regression modelling. Participants with indeterminate ILA status were excluded from these analyses. The measures of health were assigned to multivariable models adjusted for covariates including age, sex, body mass index (BMI), pack-years of smoking and current smoking status. All statistical analyses were done using R V.3.3.2.

\section{RESULTS}

The baseline characteristics of the participants in the AGES-Reykjavik cohort for which CT imaging data were available (5320 of 5764 or $92 \%$ ) are shown in table 1 . Similar to previous reports, ${ }^{3}$ people with ILA were significantly older and more often male than people without ILA. Participants with ILA were more likely to have a history of smoking, had a higher number of pack-years and were more often smokers at the time of data collection. Results regarding the associations between measures of health with ILA are shown in table 2. Associations with ILA were observed for all metrics of self-reported health and functional status. Odds of independence in ADLs were decreased among participants with ILA (OR $0.70 ; 95 \%$ CI 0.55 to 0.90 , $\mathrm{P}=0.0052)$ compared with people free of ILA (table 2). The same applied to worse health; people with ILA were significantly less likely to have good or better self-reported health status than people without ILA (OR 0.66; 95\% CI 0.52 to $0.82, \mathrm{P}=0.0003)$. People with ILA were less likely to participate in physical activity (OR 0.72 ; 95\% CI 0.56 to 0.91 , $\mathrm{P}=0.0076)$ as shown in table 2 .

\section{DISCUSSION}

These data demonstrate that ILA is associated with worse self-reported health and functional status in the AGES-Reykjavik study. This study extends previous findings ${ }^{1-3} 8$ demonstrating that, even though not diagnosed, research participants with ILA may, in some cases, share similar health outcomes as patients with ILD.

More than one reason is possible for these associations. First, it is possible that the reductions in self-reported health and functional status are being driven by the underlying, interstitial abnormalities. Another possibility is that similar underlying biological factors can cause both ILA and reduced measures of health and functional status. Lastly, it is possible that unmeasured confounders could, in part, explain some of these findings of association. These findings demonstrate that in the AGES-Reykjavik cohort, people with ILA have poorer self-reported health and reduced functional status. These results add to previous suggestions that research programmes aimed at improving treatment and prevention of ILD could benefit from directing their efforts to early stages of ILD. ${ }^{3} 9$ In conclusion, ILA are associated with measures of decreased health among elderly people.
Gisli Thor Axelsson, ${ }^{1,2}$ Rachel K Putman, ${ }^{3}$ Tetsuro Araki, ${ }^{4,5}$ Sigurdur Sigurdsson, ${ }^{1}$ Elias Freyr Gudmundsson, ${ }^{1}$ Gudny Eiriksdottir, ${ }^{1}$ Thor Aspelund, ${ }^{1,2}$ Ezra R Miller, ${ }^{3}$ Lenore J Launer, ${ }^{6}$ Tamara B Harris, ${ }^{6}$ Hiroto Hatabu, ${ }^{4,5}$ Vilmundur Gudnason, ${ }^{1,2}$ Gary Matt Hunninghake, 3,5 Gunnar Gudmundsson ${ }^{2,7}$

GTA and RKP contributed equally.

${ }^{1}$ Icelandic Heart Association, Kopavogur, Iceland ${ }^{2}$ Faculty of Medicine, University of Iceland, Reykjavik, Iceland

${ }^{3}$ Pulmonary and Critical Care Division, Brigham and Women's Hospital, Harvard Medical School, Boston, Massachusetts, USA

${ }^{4}$ Department of Radiology, Brigham and Women's Hospital, Boston, Massachusetts, USA

${ }^{5}$ Center for Pulmonary Functional Imaging, Brigham and Women's Hospital, Harvard Medical School, Boston, Massachusetts, USA

${ }^{6}$ Intramural Research Program, National Institute on Aging, Bethesda, Maryland, USA

${ }^{7}$ Department of Respiratory Medicine, Landspitali University Hospital, Reykjavik, Iceland

Correspondence to Dr Gunnar Gudmundsson, Department of Respiratory Medicine, Landspitali University Hospital, Reykjavik IS-108, Iceland; ggudmund@landspitali.is

Contributors Study design: HH, VG, GMH and GG. Acquisition, analysis or interpretation of the data: GTA, RKP, TAs, SS, EFG, GE, TAr, HH, VG, GMH and GG Statistical analysis: GTA, RKP, TAs and GMH. Obtained funding: VG, GMH and GG. All authors contributed to the critical revision of the manuscript for important intellectual content.

Funding RKP is supported by a National Institutes of Health (NIH) grant (T32 HL007633). GG is supported by a project grant from the Icelandic Research Fund (141513-051) and from the Landspitali Scientific Fund (A-2015-030, A-2016-023). The Age, Gene/ Environment Susceptibility-Reykjavik study was supported by a National Institute on Aging (NIA) grant (27120120022C), two NIH contracts (N01-AG-1-2100 and HHSN27120120022C), the NIA Intramural Research Program, Hjartavernd (the Icelandic Heart Association) and the Althingi (the Icelandic Parliament). VG is supported by an NIA grant (27120120022C) and an Icelandic Research Fund project grant (141513-051). GMH is supported by NIH grants (P01 HL114501, R01 HL111024, R01 HL135142, R01 HL130974) and a project grant from 
the Icelandic Research Fund (141513-051).

Competing interests None declared.

Ethics approval National Bioethics Committee in Iceland (VSN: 00-063) and National Institute on Ageing Intramural Institutional Review Board.

Provenance and peer review Not commissioned; externally peer reviewed.

(c) Article author(s) (or their employer(s) unless otherwise stated in the text of the article) 2018. All rights reserved. No commercial use is permitted unless otherwise expressly granted.

\section{D) Check for updates}

To cite Axelsson GT, Putman RK, Araki T, et al. Thorax 2018:73:884-886

Received 26 August 2017

Revised 11 December 2017
Accepted 18 December 2017

Published Online First 9 January 2018

Thorax 2018:73:884-886.

doi:10.1136/thoraxjnl-2017-210956

\section{REFERENCES}

1 Washko GR, Hunninghake GM, Fernandez IE, et al. Lung volumes and emphysema in smokers with interstitial lung abnormalities. N Engl J Med 2011;364:897-906.

2 Hunninghake GM, Hatabu H, Okajima Y, et al. MUC5B promoter polymorphism and interstitial lung abnormalities. N Engl J Med 2013;368:2192-200.

3 Putman RK, Hatabu H, Araki T, et al. Association between interstitial lung abnormalities and all-cause mortality. JAMA 2016;315:672-81.

4 Podolanczuk AJ, Oelsner EC, Barr RG, et al. High attenuation areas on chest computed tomography in community-dwelling adults: the MESA study. Eur Respir J 2016;48:1442-52.

5 American Thoracic Society, European Respiratory Society. American Thoracic Society/European Respiratory
Society International Multidisciplinary Consensus Classification of the Idiopathic Interstitial Pneumonias. This joint statement of the American Thoracic Society (ATS), and the European Respiratory Society (ERS) was adopted by the ATS board of directors, June 2001 and by the ERS Executive Committee, June 2001. Am J Respir Crit Care Med 2002;165:277-304.

6 Harris TB, Launer LJ, Eiriksdottir G, et al. Age, gene/environment susceptibility-Reykjavik study: multidisciplinary applied phenomics. Am J Epidemiol 2007;165:1076-87.

7 Siggeirsdottir K, Aspelund T, Jonsson BY, et al. Effect of vertebral fractures on function, quality of life and hospitalisation the AGES-Reykjavik study. Age Ageing 2012:41:351-7.

8 Doyle TJ, Washko GR, Fernandez IE, et al. Interstitial lung abnormalities and reduced exercise capacity. Am J Respir Crit Care Med 2012;185:756-62.

9 Doyle TJ, Hunninghake GM, Rosas IO. Subclinical interstitial lung disease: why you should care. Am J Respir Crit Care Med 2012;185:1147-53. 